\title{
Combining dynamical quantum typicality and numerical linked cluster expansions
}

\author{
Jonas Richter* and Robin Steinigeweg ${ }^{\dagger}$ \\ Department of Physics, University of Osnabrück, D-49069 Osnabrück, Germany
}

(Dated: March 21, 2019)

\begin{abstract}
We demonstrate that numerical linked cluster expansions (NLCE) yield a powerful approach to calculate time-dependent correlation functions for quantum many-body systems in one dimension. As a paradigmatic example, we study the dynamics of the spin current in the spin- $1 / 2 \mathrm{XXZ}$ chain for different values of anisotropy, as well as in the presence of an integrability-breaking next-nearest neighbor interaction. For short to intermediate time scales, we unveil that NLCE yields a convergence towards the thermodynamic limit already for small cluster sizes, which is much faster than in direct calculations of the autocorrelation function for systems with open or periodic boundary conditions. Most importantly, we show that the range of accessible cluster sizes in NLCE can be extended by evaluating the contributions of larger clusters by means of a pure-state approach based on the concept of dynamical quantum typicality (DQT). Even for moderate computational effort, this combination of DQT and NLCE provides a competitive alternative to existing state-of-the-art techniques, which may be applied in higher dimensions as well.
\end{abstract}

\section{INTRODUCTION}

Unraveling the complex dynamics of interacting quantum many-body systems is a central area of research of modern experimental and theoretical physics [1]. On the one hand, fascinating experiments with cold atoms $[2,3]$ and trapped ions [4] nowadays open the possibility to explore the unitary time evolution of closed quantum systems for a variety of tailored Hamiltonians and initial states. On the other hand, theoretical studies of interacting many-body systems are challenging as well, and analytic solutions are comparatively rare [5-7]. Nevertheless, much progress has been made due to the development of sophisticated numerical tools including, e.g., dynamical mean field theory [8], Krylov subspace approaches [9, 10], quantum Monte-Carlo [11], classical representations in phase space [12] or networks [13], as well as innovative machine learning implementations [14], to name just a few. While each of these methods certainly has its own specific strengths and drawbacks, their combination provides a comprehensive picture for a wide range of physical situations. Particularly for one-dimensional systems, the time-dependent density matrix renormalization group (tDMRG), including related methods based on matrix product states, are powerful techniques to study the dynamical properties of quantum systems practically in the thermodynamic limit [15-17]. However, since such approaches rely on an efficient compression of the wave function, they are generally limited by the build-up of entanglement which in turn restricts the maximum time reachable in simulations $[18,19]$.

Among the numerous methods available, exact diagonalization (ED) is arguably the most versatile approach. It can be applied to any finite-dimensional Hamiltonian, observable, and initial state. Moreover, it allows the calculation of quantum dynamics for arbitrarily long time

\footnotetext{
* jonasrichter@uos.de

$\dagger$ rsteinig@uos.de
}

scales at all temperatures [20-25]. However, ED is generally limited to rather small Hilbert-space dimensions, and this dimension grows exponentially fast for manybody problems. Substantially larger Hilbert spaces can be treated by, e.g., the concept of dynamical quantum typicality (DQT), where static and dynamic expectation values are evaluated on the basis of pure quantum states which mimic the statistical ensemble [26-36]. Both, ED and DQT, will be important building blocks of the present work. To be concrete, we will combine ED and DQT within the framework of so-called numerical linked cluster expansions (NLCE) [37]. While NLCE have been originally introduced to study quantum systems in equilibrium [38, 39] (see also Refs. [40, 41]), they also proved to be a useful approach to calculate entanglement entropies [42] and to predict steady-state properties in quantum quench scenarios [43-45] and drivendissipative systems [46]. More recently, NLCE have been successfully employed to access the full time evolution of observables resulting from a quench, with the initial state being either a (pure) product state [47, 48] or also a (mixed) thermal density matrix [49].

In this context, the present paper demonstrates that NLCE also yield a powerful approach to calculate timedependent current autocorrelations for one-dimensional quantum spin models. We particularly unveil that, on short to intermediate time scales, NLCE can outperform standard finite-size scaling in systems with open or periodic boundary conditions. Moreover, we show that NLCE can be significantly improved if the contributions of larger clusters are evaluated by means of DQT. This combination of NLCE and DQT provides a competitive alternative to existing state-of-the-art techniques operating in the thermodynamic limit.

This paper is structured as follows. In Sec. II we first introduce the model. In Sec. III we then give an overview over selected numerical methods, including ED, DQT, and NLCE, and particularly discuss the combination of DQT and NLCE. In Sec. IV this combination is applied and compared to other numerical approaches. Finally, 
we summarize and conclude in Sec. V.

\section{MODEL}

As a paradigmatic example, we consider the spin- $1 / 2$ XXZ chain, described by the Hamiltonian

$$
\mathcal{H}=J \sum_{l=1}^{L-1, L}\left(S_{l}^{x} S_{l+1}^{x}+S_{l}^{y} S_{l+1}^{y}+\Delta S_{l}^{z} S_{l+1}^{z}\right),
$$

where $S_{l}^{\mu}(\mu=x, y, z)$ are spin-1/2 operators at site $l$, $J>0$ is the antiferromagnetic coupling constant, and $\Delta$ denotes the anisotropy in the $z$ direction. Moreover, $L$ is the number of sites, and the sum in Eq. (1) runs up to $L-1(L)$ if one is interested in open (periodic) boundary conditions $\left(S_{L+1}^{\mu}=S_{1}^{\mu}\right)$. While this model is integrable in terms of the Bethe ansatz $[50,51]$, this integrability can be broken, e.g., by an additional next-nearest neighbor interaction $\Delta^{\prime} \neq 0[22,52,53]$,

$$
\mathcal{H} \rightarrow \mathcal{H}+J \sum_{l=1}^{L-2, L} \Delta^{\prime} S_{l}^{z} S_{l+2}^{z} .
$$

Since the total magnetization $M=\sum_{l} S_{l}^{z}$ is conserved for all values of $\Delta$ and $\Delta^{\prime}$, the spin current is well-defined and has the well-known form [54]

$$
j=J \sum_{l=1}^{L-1, L}\left(S_{l}^{x} S_{l+1}^{y}-S_{l}^{y} S_{l+1}^{x}\right),
$$

which also depends on the specific boundary condition chosen. In this paper, we explore the time dependence of the current autocorrelation function

$$
\langle j(t) j\rangle_{\mathrm{eq}}=\operatorname{Tr}\left[j(t) j \rho_{\mathrm{eq}}\right],
$$

where $\rho_{\text {eq }}=e^{-\beta \mathcal{H}} / \mathcal{Z}_{\text {eq }}$ is the canonical ensemble at inverse temperature $\beta=1 / T$, and $j(t)=e^{i \mathcal{H} t} j e^{-i \mathcal{H} t}$. Within linear response theory (LRT), $\langle j(t) j\rangle_{\text {eq }}$ is directly related to transport properties via the Kubo formula. For earlier studies of current autocorrelations in the spin1/2 XXZ chain see, e.g., Refs. [18, 36, 55-59]. Note further that there exist of course also other approaches to transport in low-dimensional quantum spin systems apart from LRT [60-62].

\section{NUMERICAL APPROACH}

Before discussing NLCE below in detail, it is instructive to briefly reiterate how to calculate time-dependent correlation functions such as $\langle j(t) j\rangle_{\text {eq }}$ by ED and DQT.

\section{A. Exact diagonalization}

Upon diagonalizing $\mathcal{H}$ for finite $L$, the full knowledge of eigenstates and eigenenergies in principle allows for the computation of all static and dynamic properties. In this context, $\langle j(t) j\rangle_{\text {eq }}$ is conveniently written in a spectral representation,

$$
\langle j(t) j\rangle_{\text {eq }}=\frac{1}{\mathcal{Z}_{\text {eq }}} \sum_{m, n} e^{-\beta E_{m}} e^{-i\left(E_{n}-E_{m}\right) t}|\langle m|j| n\rangle|^{2},
$$

where the sum runs over all eigenstates $|m\rangle,|n\rangle$ of $\mathcal{H}$ with respective eigenenergies $E_{m}, E_{n}$. Due to the exponential growth of the Hilbert space, however, ED is limited to rather small system sizes. This limitation becomes particularly severe in the case of open boundary conditions where translation symmetry cannot be exploited. Nevertheless, ED for small systems will be a major cornerstone for NLCE.

\section{B. Dynamical quantum typicality}

For system sizes outside the range of ED, the method of DQT [26-36] has been established as a very useful numerical approach. This method relies on the fact that even a single pure state can have the same properties as the full statistical ensemble. Specifically, $\langle j(t) j\rangle_{\text {eq }}$ can be written as a simple scalar product with two (auxiliary) pure states $\left|\psi_{\beta}(t)\right\rangle$ and $\left|\varphi_{\beta}(t)\right\rangle,[34,36,59]$

$$
\langle j(t) j\rangle_{\mathrm{eq}}=\frac{\left\langle\psi_{\beta}(t)|j| \varphi_{\beta}(t)\right\rangle}{\left\langle\psi_{\beta}(0) \mid \psi_{\beta}(0)\right\rangle}+\epsilon,
$$

$\left|\psi_{\beta}(t)\right\rangle=e^{-i \mathcal{H} t} e^{-\beta \mathcal{H} / 2}|\psi\rangle,\left|\varphi_{\beta}(t)\right\rangle=e^{-i \mathcal{H} t} j e^{-\beta \mathcal{H} / 2}|\psi\rangle$, and the reference pure state $|\psi\rangle$ is drawn at random from the full Hilbert space according to the unitary invariant Haar measure [30]. Importantly, the statistical error $\epsilon=\epsilon(|\psi\rangle)$ has zero mean, $\bar{\epsilon}=0$, and its standard deviation scales as $\sigma(\epsilon) \propto 1 / \sqrt{d_{\text {eff }}}$, where $d_{\text {eff }}=\mathcal{Z}_{\text {eq }} / e^{-\beta E_{0}}$ is the effective dimension of the Hilbert space and $E_{0}$ is the ground-state energy of $\mathcal{H}[30,31,34,36,59]$. Thus, $\sigma(\epsilon)$ decreases exponentially with increasing $L$ and, for many practical purposes, $\epsilon$ can be neglected for mediumsized systems already (especially for $\beta \rightarrow 0$ ). However, if one wants to improve the accuracy of the DQT approximation even further, it is of course always possible to evaluate (nominator and denominator of) Eq. (6) as an average over $N_{S}$ independent realizations of the random pure state $|\psi\rangle[32,63]$. In fact, such an averaging turns out to be important when NLCE is combined with DQT as done below.

The main advantage of Eq. (6) comes from the fact that the time evolution of pure states can be generated by iteratively solving the Schrödinger equation. To this end, various sophisticated methods are available such as, e.g., Trotter decompositions [64], Chebychev polynomials $[65,66]$, Krylov subspace techniques [9, 67], and RungeKutta schemes $[34,36]$. A unifying feature of all these methods is that they essentially require the calculation of matrix-vector products, which can be implemented both time- and memory-efficient due to the sparseness 
of the involved operators. Thus, no diagonalization of $\mathcal{H}$ is needed and Eq. (6) can be evaluated for Hilbert-space dimensions substantially larger compared to ED.

\section{Numerical linked cluster expansions}

Let us now come to NLCE. Note that we intentionally refrain from a general introduction to NLCE, for detailed explanations see, e.g., Ref. [37]. Instead, we choose to sketch more specifically how NLCE can be used to obtain current autocorrelations in a one-dimensional geometry. Within NLCE, an extensive quantity per lattice site is calculated as the sum of contributions from all connected clusters which can be embedded on the lattice,

$$
\langle j(t) j\rangle_{\mathrm{eq}} / L=\sum_{c} \mathcal{L}_{c} W_{c}(t),
$$

where $W_{c}(t)$ is the weight of cluster $c$ with multiplicity $\mathcal{L}_{c}$. While the identification of all linked clusters for a given (arbitrary) lattice can be a cumbersome procedure, this identification becomes straightforward in one dimension. Given an infinitely long and translational-invariant chain, the linked clusters are just chains as well, which comprise a certain (finite) number of sites. Moreover, for a fixed cluster size, there exists only a single topologically distinct cluster $c$ (since any translation of $c$ is just equivalent to $c$ ), cf. [68]. Therefore, we have $\mathcal{L}_{c}=1$ in Eq. (7) and we can identify the cluster index $c$ as the number of sites in the respective cluster. The weights $W_{c}(t)$ in Eq. (7) are calculated by the so-called inclusion-exclusion principle,

$$
W_{c}(t)=\langle j(t) j\rangle_{\mathrm{eq}}^{(c)}-\sum_{s \subset c} W_{s}(t),
$$

where $\langle j(t) j\rangle_{\mathrm{eq}}^{(c)}$ denotes the current autocorrelation evaluated on the cluster $c$, and the sum runs over all subclusters of $c$. Due to the definition of $j$ in Eq. (3), the smallest nontrivial cluster which needs to be considered is a single bond connecting just two lattice sites. The weight of this cluster then follows as $W_{2}(t)=\langle j(t) j\rangle_{\mathrm{eq}}^{(2)}$, since there are no subclusters in this case. A cluster of length $c=3$ obviously has only two linked subclusters of length $c=2$, i.e., $W_{3}(t)=\langle j(t) j\rangle_{\mathrm{eq}}^{(3)}-2 W_{2}(t)$. Generalizing this scheme, the weight for $c \geq 3$ reads

$$
W_{c}(t)=\langle j(t) j\rangle_{\mathrm{eq}}^{(c)}-\sum_{s=2}^{c-1}(c-s+1) W_{s}(t) .
$$

To summarize, $\langle j(t) j\rangle_{\mathrm{eq}} / L$ in the thermodynamic limit $L \rightarrow \infty$ is calculated as a sum over weights $W_{c}(t)$ and the calculation of these weights requires the evaluation of $\langle j(t) j\rangle_{\text {eq }}^{(c)}$ on clusters with increasing size $c=2,3, \ldots$ and open boundary conditions.

In practice, however, it is only possible to consider contributions of clusters which are small enough to be
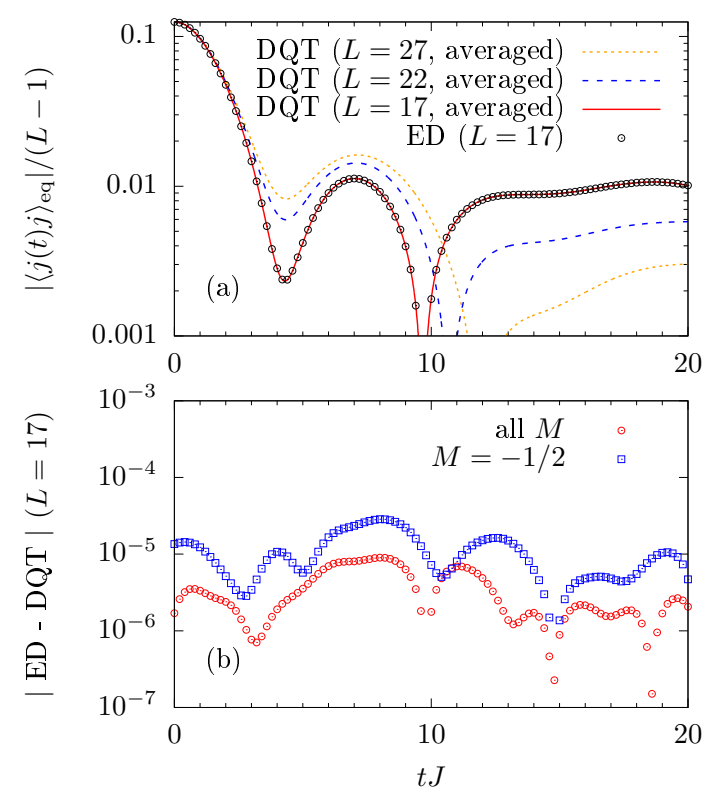

FIG. 1. (Color online) (a) $\left|\langle j(t) j\rangle_{\text {eq }}\right| /(L-1)$ for $L=17$ (ED) and $L=17,22,27$ (DQT) for systems with open boundary conditions. (b) Absolute difference between ED and DQT $(L=17)$. Averaging over all magnetization sectors, as done in this paper, improves the accuracy further. Other parameters: $\Delta=1, \Delta^{\prime}=0$, and $\beta=0$.

treated numerically, and the sum in Eq. (7) eventually has to be truncated to a maximum cluster size $C$. On the one hand, for NLCE implementations of thermodynamic quantities, a larger value of $C$ often improves the convergence of the expansion down to lower temperatures [69]. On the other hand, for time-dependent quantities, the value of $C$ will directly correspond to the time scale on which the NLCE can yield reliable results, cf. Ref. [47]. Thus, it is generally highly desirable to include clusters as large as possible. In this paper, we demonstrate that NLCE can be significantly improved if it is combined with DQT in order to evaluate larger clusters outside the range of ED.

Remarkably, in the present one-dimensional situation, it is straightforward to show that a truncation of Eq. (7) to order $C$ takes on the simple form

$$
\sum_{c=2}^{C} W_{c}(t)=\langle j(t) j\rangle_{\mathrm{eq}}^{(C)}-\langle j(t) j\rangle_{\mathrm{eq}}^{(C-1)},
$$

and is just the difference between the two largest clusters $C$ and $C-1$. Since this difference might be sensitive to numerical inaccuracies, it is in this context convenient to average the DQT calculations over $N_{S}$ independent random states. We here choose $N_{S} \times 2^{c}>5000 \times 2^{17}$, see also the table in Appendix C. 

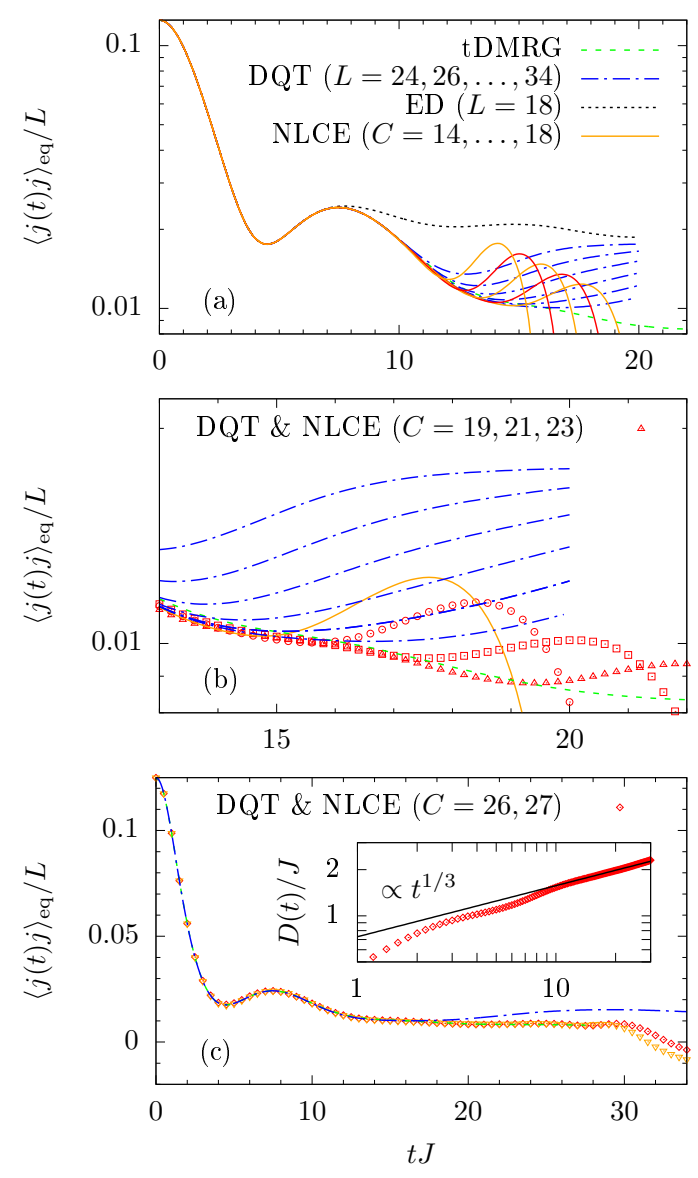

FIG. 2. (Color online) $\langle j(t) j\rangle_{\text {eq }} / L$ for $\Delta=1, \Delta^{\prime}=0$, and $\beta=$ 0 . (a) NLCE for $C=14, \ldots, 18$, compared to ED, DQT [36, 59, 70], and tDMRG [58]. (b) Combination DQT \& NLCE for $C=19,21,23$. (c) Linear plot with data for $C=26,27$. Inset: Diffusion coefficient $D(t)$ for $C=27$ and a power law $\propto t^{1 / 3}$ as a guide to the eye.

\section{APPLICATION}

We start with discussing the accuracy of the pure-state approach in Eq. (6). Thus, Fig. 1 (a) exemplarily shows $\left|\langle j(t) j\rangle_{\text {eq }}\right|$ for $\Delta=1, \Delta^{\prime}=0$, and $\beta=0$, calculated by ED and DQT for open boundary conditions and $L=17$. In the semilogarithmic plot, one finds that both methods agree nicely with each other, indicating that the statistical error $\epsilon$ in Eq. (6) is indeed very small. In addition, we depict DQT data for $L=22,27$, which visualize that finite-size effects become non-negligible already for times $t J \gtrsim 5$. Note that ED is already unfeasible for these $L$ such that no comparison is possible. In order to quantify $\epsilon$, Fig. 1 (b) shows the absolute difference between ED and DQT for $L=17$. For all times depicted, we find $\epsilon=\mathcal{O}\left(10^{-5}-10^{-6}\right)$ when using $N_{S}=5000$.

We now turn to our NLCE results, focusing on the integrable case $\Delta^{\prime}=0$ at infinite temperature $\beta=0$. In Fig. $2(\mathrm{a}),\langle j(t) j\rangle_{\text {eq }} / L$ is shown at the isotropic point
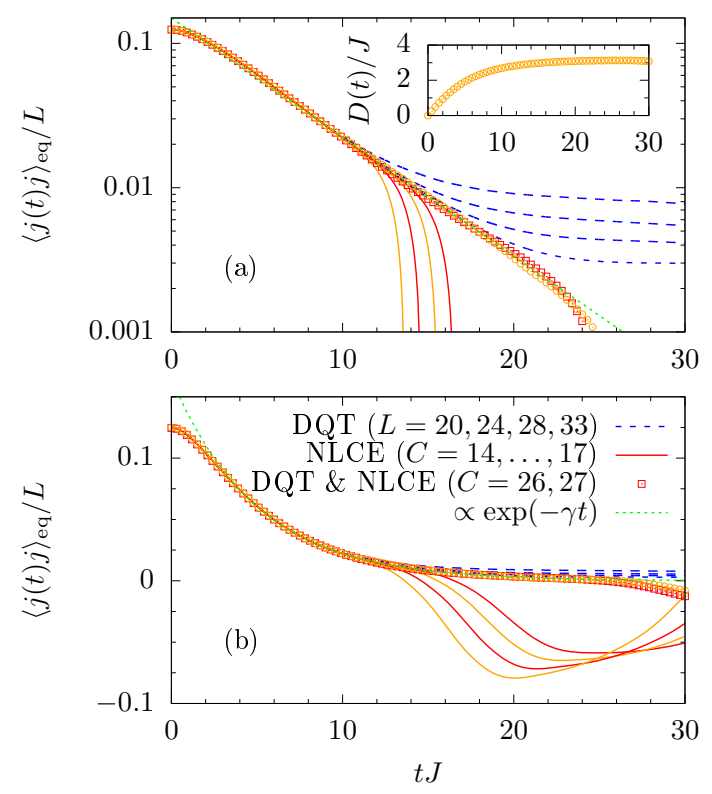

FIG. 3. (Color online) $\langle j(t) j\rangle_{\text {eq }} / L$ for $\Delta=\Delta^{\prime}=0.5$ and $\beta=0$, obtained from $\operatorname{NLCE}(C=14, \ldots, 17)$ and DQT \& NLCE $(C=26,27)$ in a (a) semilogarithmic and (b) linear plot. An exponential is shown as a guide to the eye. Inset: Diffusion coefficient $D(t)$ for $C=27$.

$\Delta=1$, in a semilogarithmic plot. On the one hand, we depict NLCE data for $C=14, \ldots, 18$. On the other hand, this data is compared to data for systems with periodic boundary conditions, obtained by either ED $(L=18)$ or DQT $(L=24,26, \ldots, 34)[36,59]$, and to tDMRG data [58]. We find that NLCE converges up to a maximum time, increasing with $C$, until the expansion eventually breaks down. Remarkably, however, one can clearly see that NLCE is converged for a substantially longer time compared to ED, which becomes particularly evident when comparing NLCE for $C=18$ and ED for $L=18$. Moreover, NLCE for $C=18$ coincides with tDMRG up to a time $t J \lesssim 15$, which approximately corresponds to the convergence reached in DQT with $L=34$. This observation is particularly remarkable since the largest cluster in an expansion up to $C=18$ also consists of $L=18$ sites only. Thus, on short to intermediate times, NLCE clearly outperforms standard finite-size scaling.

Next, Fig. 2 (b) shows a close-up of the data for intermediate times $13 \leq t J \leq 22$. Furthermore, we now depict NLCE for larger $C=19,21,23$, which can be obtained thanks to the combination DQT \& NLCE. For $t J \leq 15$, one observes that all expansion orders lie on top of each other, demonstrating that DQT is indeed accurate enough to be combined with NLCE. Moreover, we find that NLCE continues to follow the tDMRG for longer and longer times when $C$ is increased. In fact, as can be seen in the linear plot in Fig. 2 (c), NLCE for $C=27$ essentially agrees with tDMRG up to times 
$t J \lesssim 27$, in contrast to DQT data for $L=34$ [70], which exhibits visible finite-size effects. Note that it is in principle possible to calculate even larger $c>27$ [71].

Eventually, the inset in Fig. 2 (c) shows the diffusion coefficient [55]

$$
D(t)=\frac{1}{\chi} \int_{0}^{t} \frac{\left\langle j\left(t^{\prime}\right) j\right\rangle}{L} \mathrm{~d} t^{\prime}, \quad \chi=\frac{1}{4}
$$

for the largest expansion order $C=27$. After $t J \sim 10$, $D(t)$ is consistent with a power-law scaling $\propto t^{1 / 3}$ and thus superdiffusive transport. This specific exponent has been recently suggested in Ref. [73] (see also [62]), which cannot be seen in ED of small systems.

Finally, we are going to discuss NLCE for a nonintegrable model as well. In Fig. 3 (a), we show $\langle j(t) j\rangle_{\text {eq }} / L$ for $\Delta=\Delta^{\prime}=0.5$. Once again we compare NLCE for various $C$ to data for periodic chains of finite length [53]. As a guide to the eye, we depict an exponential $\propto \exp (-\gamma t)$, which describes the decay process reasonably well $[74,75]$. Similarly to Fig. 2, we find that NLCE outperforms standard finite-size scaling on short to medium time scales, in the sense that NLCE converges fast to the exponential even for small $C$. However, since finite-size effects are typically smaller for nonintegrable models, the advantage of NLCE becomes less pronounced compared to the integrable case shown in Fig. 2.

\section{CONCLUSION}

To summarize, we have shown that NLCE is a powerful approach to dynamics in a one-dimensional geometry, particularly when it is additionally combined with DQT. This we have done by comparing to existing results from various state-of-the-art methods. While we have focused on two case studies, the combination DQT \& NLCE yields equally convincing results for a wider choice of parameters and also at finite temperature $\beta>0$ (see Appendix A and B for details).

Promising directions of future research also include the application of the DQT \& NLCE combination to other dynamical quantities in one or two spatial dimensions. In particular, the extension of NLCE to larger cluster sizes is of direct relevance to the study of quench dynamics starting from thermal initial states, cf. Ref. [49]. Such classes of initial states have been shown to be amenable to the concept of typicality as well $[76,77]$.

\section{Acknowledgments}

We are grateful to C. Karrasch for sending us tDMRG data for comparison as well as to F. Heidrich-Meisner, L. Vidmar, J. Herbrych, and J. Gemmer for fruitful discussions. This work has been funded by the Deutsche Forschungsgemeinschaft (DFG) - STE 2243/31; 397067869; 355031190 - within the DFG Research Unit FOR 2692.
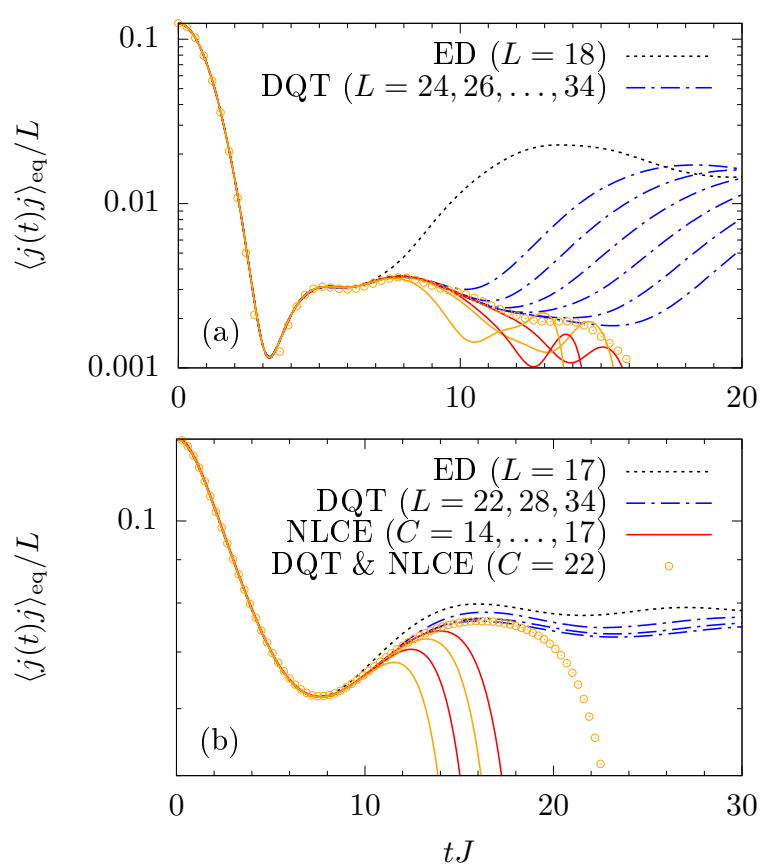

FIG. 4. (Color online) Current autocorrelation $\langle j(t) j\rangle_{\text {eq }} / L$ at $\beta=0$ and anisotropy (a) $\Delta=1.5, \Delta^{\prime}=0$; (b) $\Delta=0.5$, $\Delta^{\prime}=0$. NLCE and combination DQT \& NLCE with different expansion order $C$, compared to ED and DQT calculations for finite systems with periodic boundary conditions [36, 59].

\section{Appendix A: Current autocorrelations with NLCE for anisotropies $\Delta \neq 1$}

In the main part of this paper, we have mostly focused on the isotropic Heisenberg chain with $\Delta=1$. In order to substantiate our findings even further, let us now present NLCE results also for other anisotropies $\Delta \neq 1$. In Fig. $4,\langle j(t) j\rangle_{\mathrm{eq}} / L$ is shown for $\Delta=1.5$ and $\Delta=0.5$. In both cases, we again compare NLCE for expansion or-

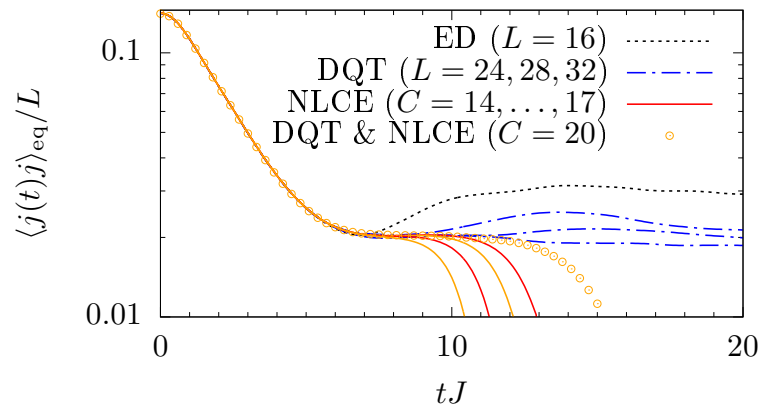

FIG. 5. (Color online) Current autocorrelation $\langle j(t) j\rangle_{\text {eq }} / L$ at finite temperature $\beta J=1$ for $\Delta=1, \Delta^{\prime}=0$. NLCE and combination DQT \& NLCE with different expansion order $C$, compared to ED and DQT calculations for finite systems with periodic boundary conditions [36, 59]. 
TABLE I. Number of random states $N_{S}$ for a given system size $L$, as used in the DQT calculations underlying the NLCE for $\Delta=1, \Delta^{\prime}=0$, and $\beta=0$ in Figs. 2 (b) and (c). The product $N_{S} \times 2^{L}$ is always larger than $5000 \times 2^{17}$.

\begin{tabular}{|r|r|c|}
\hline$L$ & $N_{S}$ & $N_{S} \times 2^{L}$ \\
\hline \hline 17 & 5000 & $6.55 \times 10^{8}$ \\
\hline \hline 19 & 7900 & $41.4 \times 10^{8}$ \\
20 & 3725 & $39.1 \times 10^{8}$ \\
21 & 1260 & $26.4 \times 10^{8}$ \\
22 & 643 & $27.0 \times 10^{8}$ \\
23 & 298 & $25.0 \times 10^{8}$ \\
24 & 85 & $14.3 \times 10^{8}$ \\
25 & 44 & $14.8 \times 10^{8}$ \\
26 & 34 & $22.8 \times 10^{8}$ \\
27 & 8 & $10.7 \times 10^{8}$ \\
\hline
\end{tabular}

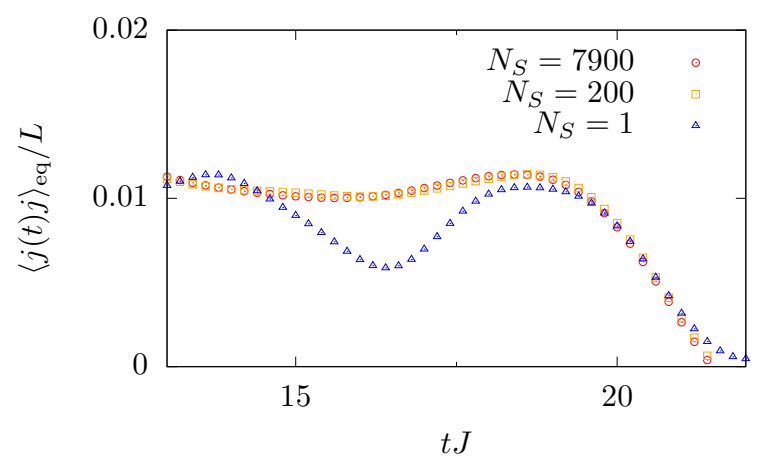

FIG. 6. (Color online) Expansion order $C=19$ according to the combination DQT \& NLCE, as shown in Fig. 2 (b) in the main text, but now for a different number of random states: $N_{S}=1,200$, and 7900. While the curves for $N_{S}=200$ and 7900 are practically the same, $N_{S}=1$ clearly differs for such a small $C$.

$\operatorname{der} C=14, \ldots, 17$ with ED and DQT for periodic chains of finite length. Comparing small and large $\Delta$, we find that the convergence of the NLCE is a little slower in the case of $\Delta=1.5$, where the expansion also exhibits evenodd-like effects. This might be caused by the fact that the autocorrelation function takes on very small values for this choice of $\Delta$. Generally, however, the situation for $\Delta=1.5$ and $\Delta=0.5$ is very similar compared to the isotropic case discussed in the main part of this paper, i.e., NLCE for given expansion order is converged to the thermodynamic limit for a longer time scale than in corresponding calculations of systems with periodic boundaries.

\section{Appendix B: Current autocorrelations with NLCE for finite temperatures}

Eventually, let us demonstrate that NLCE is certainly not restricted to the infinite-temperature limit. To this end, Fig. 5 exemplarily shows $\langle j(t) j\rangle_{\text {eq }} / L$ at the finite temperature $\beta J=1$ for the single choice $\Delta=1$. We find that, already for $C=20$, NLCE is able to see a constant plateau up to times $t J \lesssim 12$, which is clearly missed by ED for $L=16$ and only captured by DQT for significantly larger systems with $L=28,32$. Thus, we conclude that NLCE also provides a powerful approach to current autocorrelations for a wider range of temperatures.

\section{Appendix C: Number of random states}

In Tab. I, we specify the number of random states $N_{S}$ for a given system size $L$, as used in the DQT calculations underlying the NLCE for $\Delta=1, \Delta^{\prime}=0$, and $\beta=0$ in Figs. 2 (b) and (c). As stated before, the product $N_{S} \times 2^{L}$ is always larger than $5000 \times 2^{17}$. While it is evident from the comprehensive comparison in the main text that this averaging is sufficient, it might also be insightful to see that averaging is indeed important for small expansion orders $C$. We therefore depict in Fig. 6 the expansion or$\operatorname{der} C=19$ according to the combination DQT \& NLCE, as shown in Fig. 2 (b), but now for a different number of random states: $N_{S}=1,200$, and 7900 . While the curves for $N_{S}=200$ and 7900 are practically the same, $N_{S}=1$ clearly differs for such a small $C$.
[1] J. Eisert, M. Friesdorf, and C. Gogolin, Nature Phys. 11, 124 (2015).

[2] I. Bloch, J. Dalibard, and S. Nascimbène, Nat. Phys. 8, 267 (2012).

[3] T. Langen, R. Geiger, and J. Schmiedmayer, Ann. Rev. Condens. Matter Phys. 6, 201 (2015).

[4] R. Blatt and C. F. Roos, Nat. Phys. 8, 277 (2012).

[5] F. H. L. Essler and M. Fagotti, J. Stat. Mech. 2016, 064002 (2016).

[6] O. A. Castro-Alvaredo, B. Doyon, and T. Yoshimura, Phys. Rev. X 6, 041065 (2016).
[7] B. Bertini, M. Collura, J. De Nardis, and M. Fagotti, Phys. Rev. Lett. 117, 207201 (2016).

[8] H. Aoki, N. Tsuji, M. Eckstein, M. Kollar, T. Oka, and P. Werner, Rev. Mod. Phys. 86, 779 (2014).

[9] A. Nauts and R. E. Wyatt, Phys. Rev. Lett. 51, 2238 (1983).

[10] M. W. Long, P. Prelovšek, S. El Shawish, J. Karadamoglou, and X. Zotos, Phys. Rev. B 68, 235106 (2003).

[11] F. Goth and F. F. Assaad, Phys. Rev. B 85, 085129 (2012). 
[12] J. Wurtz, A. Polkovnikov, and D. Sels, Ann. Phys. 395, 341 (2018).

[13] M. Schmitt and M. Heyl, SciPost Phys. 4, 013 (2018).

[14] G. Carleo and M. Troyer, Science 355, 602 (2017).

[15] U. Schollwöck, Rev. Mod. Phys. 77, 259 (2005); Ann. Phys. 326, 96 (2011).

[16] G. Vidal, Phys. Rev. Lett. 93, 040502 (2004).

[17] S. R. White and A. E. Feiguin, Phys. Rev. Lett. 93, 076401 (2004).

[18] J. Sirker, R. G. Pereira, and I. Affleck, Phys. Rev. Lett. 103, 216602 (2009).

[19] D. M. Kennes and C. Karrasch, Comp. Phys. Comm. 200, 37 (2016).

[20] A. W. Sandvik, AIP Conf. Proc. 1297, 135 (2010).

[21] B. N. Narozhny, A. J. Millis, and N. Andrei, Phys. Rev. B 58, R2921(R) (1998).

[22] F. Heidrich-Meisner, A. Honecker, D. C. Cabra, and W. Brenig, Phys. Rev. B 68, 134436 (2003).

[23] R. Steinigeweg and W. Brenig, Phys. Rev. Lett. 107, 250602 (2011).

[24] E. J. Torres-Herrera and L. F. Santos, Phys. Rev. B 92, 014208 (2015).

[25] M. Schmitt and S. Kehrein, Phys. Rev. B 98, 180301(R) (2018).

[26] J. Gemmer, M. Michel, and G. Mahler, Quantum Thermodynamics (Springer, Berlin, 2004).

[27] S. Popescu, A. J. Short, and A. Winter, Nat. Phys. 2, 754 (2006).

[28] S. Goldstein, J. L. Lebowitz, R. Tumulka, and N. Zanghì, Phys. Rev. Lett. 96, 050403 (2006).

[29] P. Reimann, Phys. Rev. Lett. 99, 160404 (2007).

[30] C. Bartsch and J. Gemmer, Phys. Rev. Lett. 102, 110403 (2009).

[31] A. Hams and H. De Raedt, Phys. Rev. E 62, 4365 (2000).

[32] T. Iitaka and T. Ebisuzaki, Phys. Rev. Lett. 90, 047203 (2003).

[33] S. Sugiura and A. Shimizu, Phys. Rev. Lett. 111, 010401 (2013).

[34] T. A. Elsayed and B. V. Fine, Phys. Rev. Lett. 110, 070404 (2013).

[35] T. Monnai and A. Sugita, J. Phys. Soc. Jpn. 83, 094001 (2014).

[36] R. Steinigeweg, J. Gemmer, and W. Brenig, Phys. Rev. Lett. 112, 120601 (2014).

[37] B. Tang, E. Khatami, and M. Rigol, Comput. Phys. Commun. 184, 557 (2013).

[38] M. Rigol, T. Bryant, and R. R. P. Singh, Phys. Rev. Lett. 97, 187202 (2006).

[39] M. Rigol, T. Bryant, and R. R. P. Singh, Phys. Rev. E 75, 061118 (2007).

[40] E. Khatami and M. Rigol, Phys. Rev. B 83, 134431 (2011).

[41] D. Ixert, T. Tischler, and K. P. Schmidt, Phys. Rev. B 92, 174422 (2015).

[42] A. B. Kallin, K. Hyatt, R. R. P. Singh, and R. G. Melko, Phys. Rev. Lett. 110, 135702 (2013).

[43] M. Rigol, Phys. Rev. Lett. 112, 170601 (2014).

[44] B. Wouters, J. De Nardis, M. Brockmann, D. Fioretto, M. Rigol, and J.-S. Caux, Phys. Rev. Lett. 113, 117202 (2014).

[45] K. Mallayya and M. Rigol, Phys. Rev. E 95, 033302 (2017).
[46] A. Biella, J. Jin, O. Viyuela, C. Ciuti, R. Fazio, and D. Rossini, Phys. Rev. B 97, 035103 (2018).

[47] I. G. White, B. Sundar, and K. R. A. Hazzard, arXiv:1710.07696.

[48] E. Guardado-Sanchez, P. T. Brown, D. Mitra, T. Devakul, D. A. Huse, P. Schauß, and W. S. Bakr, Phys. Rev. X 8, 021069 (2018).

[49] K. Mallayya and M. Rigol, Phys. Rev. Lett. 120, 070603 (2018).

[50] X. Zotos, Phys. Rev. Lett. 82, 1764 (1999).

[51] A. Klümper and K. Sakai, J. Phys. A 35, 2173 (2002).

[52] R. Steinigeweg, J. Herbrych, and P. Prelovšek, Phys. Rev. E 87, 012118 (2013).

[53] J. Richter, F. Jin, H. De Raedt, K. Michielsen, J. Gemmer, and R. Steinigeweg, Phys. Rev. B 97, 174430 (2018).

[54] F. Heidrich-Meisner, A. Honecker, and W. Brenig, Eur. Phys. J. Special Topics 151, 135 (2007).

[55] R. Steinigeweg and J. Gemmer, Phys. Rev. B 80, 184402 (2009).

[56] C. Karrasch, J. H. Bardarson, and J. E. Moore, Phys. Rev. Lett. 108, 227206 (2012).

[57] C. Karrasch, J. Hauschild, S. Langer, and F. HeidrichMeisner, Phys. Rev. B 87, 245128 (2013).

[58] C. Karrasch, D. M. Kennes, and F. Heidrich-Meisner, Phys. Rev. B 91, 115130 (2015).

[59] R. Steinigeweg, J. Gemmer, and W. Brenig, Phys. Rev. B 91, 104404 (2015).

[60] M. Michel, O. Hess, H. Wichterich, and J. Gemmer, Phys. Rev. B 77, 104303 (2008).

[61] T. Prosen and M. Žnidarič, J. Stat. Mech. 2009, P02035 (2009).

[62] M. Ljubotina, M. Žnidarič, and T. Prosen, Nat. Comm. 8, 16117 (2017).

[63] I. Rousochatzakis, S. Kourtis, J. Knolle, R. Moessner, and N. B. Perkins, arXiv:1811.01671.

[64] H. De Raedt and K. Michielsen, in Handbook of Theoretical and Computational Nanotechnology (American Scientific Publishers, Los Angeles, 2006).

[65] V. V. Dobrovitski and H. De Raedt, Phys. Rev. E 67, 056702 (2003).

[66] A. Weiße, G. Wellein, A. Alvermann, and H. Fehske, Rev. Mod. Phys. 78, 275 (2006).

[67] V. K. Varma, A. Lerose, F. Pietracaprina, J. Goold, and A. Scardicchio, J. Stat. Mech. 2017053101 (2017).

[68] For chains with next-nearest neighbor interaction $\Delta^{\prime} \neq 0$, there are in principle more than one topologically distinct cluster for a fixed cluster size $c$, cf. Ref. [45]. However, also in this case, the NLCE can be organized with maximally connected clusters such that Eq. (9) remains valid.

[69] K. Bhattaram and E. Khatami, arXiv:1810.06202.

[70] While DQT data for $L=34$ can already be found in Ref. [59], we here recalculate data for even longer times.

[71] Note that clusters with $c \sim 40$ lattice sites can be treated numerically using supercomputers [72].

[72] J. Richter, F. Jin, L. Knipschild, J. Herbrych, H. De Raedt, K. Michielsen, J. Gemmer, and R. Steinigeweg, arXiv:1811.02806.

[73] S. Gopalakrishnan and R. Vasseur, arXiv:1812.02701.

[74] R. Steinigeweg, Phys. Rev. E 84, 011136 (2011).

[75] J. Herbrych, R. Steinigeweg, and P. Prelovšek, Phys. Rev. B 86, 115106 (2012).

[76] J. Richter, J. Herbrych, and R. Steinigeweg, Phys. Rev. B 98, 134302 (2018).

[77] J. Richter, J. Gemmer, and R. Steinigeweg, arXiv:1805.11625. 